\title{
À quels territoires s'intéressent les agronomes ? Le point de vue d'un géographe tropicaliste
}

\author{
Patrick Caron \\ Géographe, Département Territoires, Environnement et Acteurs (TERA), CIRAD, 73 rue J.-F. Breton, TA 60/15, \\ 34398 Montpellier cedex 5, France
}

Comment les agronomes français en sont-ils arrivés à s'intéresser à la notion de territoire? Partant de recherches conduites en milieu tropical, l'auteur distingue différentes attitudes utilisées par les agronomes face à cette notion. Mais, au-delà de la diversité des approches, son interrogation met en évidence la nécessité d'un projet scientifique qui permette à l'agronomie de renouer avec sa vocation de science intégrative.

La Rédaction

\section{Mots-clés :}

agronomie ; territoire : développement territorial ; science d'intégration

\section{Keywords:}

agronomy;

territory;

territorial development; integrative science
Résumé - L'auteur identifie différentes voies développées par les agronomes pour traiter et agir sur les territoires. Avant cela, il précise ce qu'il entend par territoire et revient sur les racines et les évolutions intellectuelles qui ont poussé les agronomes au cours des trente dernières années à s'y intéresser. À partir d'illustrations non exhaustives portant sur des recherches conduites en milieu tropical, quatre voies sont présentées comme autant de manières de s'intéresser au territoire : (i) agronomie de l'écosystème régional ; (ii) agronomie du fait technique à l'échelle territoriale ; (iii) agronomie territoriale ; (iv) agronomie intégratrice. Le débat sur la prise en compte du territoire illustre les controverses qui s'expriment au sein de la discipline à propos d'une recomposition des identités. Ce qui fait territoire ne va pas de soi, même s'il ne fait pas débat pour autant. Assumer la pluralité des pensées et des méthodes apparaît nécessaire pour que les agronomes se situent et traitent les problèmes liés à la gestion des ressources sur un territoire. De nouveaux approfondissements s'avèrent nécessaires pour renouer avec le dessein brouillé d'une science d'intégration.

\begin{abstract}
What kind of territories are agronomists interested in? A tropical geographer's perspective. Following a brief definition of the concept of territory as used here and an analysis of the intellectual roots and reasons that motivated the agronomists' interest in it, the author identifies different ways of analysing and acting upon territories. Based on examples from tropical agricultural research, P. Caron outlines four approaches to the territory: (i) regional ecosystem agronomy, (ii) agronomy focusing on technical issues at the territory level, (iii) territorial agronomy, (iv) integrative agronomy. The debates on the ways of taking the territory into account highlight the difficult identity transformation within agronomy. What is defined as territory is not self-evident, but is not often debated. Recognition of the diversity of paradigms and methods by agronomists is a necessary step towards dealing with issues linked to the management of territorial resources. Further work is needed to recapture the objective of agronomy as an integrative science.
\end{abstract}

Le concept de territoire jouit aujourd'hui d'un prestige certain. On élargit ainsi, pour qui s'intéresse au secteur agricole, le champ des préoccupations en resituant ce secteur et ses contributions à la transformation des espaces ruraux. On se donne les moyens de comprendre les attentes de la société et les fonctions assignées à

Auteur correspondant : caron@cirad.fr l'agriculture, en particulier dans le domaine de l'environnement. Le territoire est à la mode, tant et si bien qu'il a fait irruption dans les textes de politique agricole. Cet engouement masque cependant une diversité d'acceptions et des difficultés à communiquer (Lévy et Lussault, 2003). Le terme est, par exemple, difficilement traduisible en anglais, ce qui pose des problèmes de compréhension au sein de la communauté scientifique internationale. 
Inspirée de Brunet et al. (1992), la définition retenue ici s'appuie sur les principaux éléments suivants : (i) un espace borné, aux limites plus ou moins précises, et approprié par un groupe social; (ii) un sentiment ou une conscience d'appartenance de la part de ses habitants ; (iii) l'existence de formes d'autorité politique et de règles d'organisation et de fonctionnement.

Le territoire, construit social dont les attributs résultent de cheminements historiques spécifiques, se présente donc comme un cadre d'action individuelle, collective et publique, et d'adaptation des acteurs aux évolutions de leur environnement. Représentation caractérisée par une unité spatiale (Bonnemaison, 2000), il n'en n'a pas moins une base réticulaire. Il repose, entre autres, sur l'existence d'acteurs et de réseaux sociaux qui en assurent la construction, la défense, la promotion, l'évolution, la reproduction, la gestion, l'usage et le partage des ressources et richesses. Mais, ressource mobilisable, il est aussi processeur de changement. Défini ainsi, il est porteur de développement territorial : il permet d'imaginer la conception de nouvelles actions, visant à accroître la maîtrise par les acteurs des processus de changement, d'anticipation et de réponses aux aléas, de minimisation des risques et des incertitudes par une meilleure capacité d'apprentissage (Camagni, 1991). C'est ce qui intéresse certains agronomes et représente, pour eux, un concept opératoire.

Cet intérêt se double d'une volonté souvent implicite de prendre en compte, pour comprendre ou pour agir, de nombreux acteurs, aux intérêts différents et parfois conflictuels, et de multiples niveaux fonctionnels interdépendants, territoriaux, sectoriels ou écologiques. Cela conduit, entre autres, à reconnaître une multiplicité de territoires aux contours, formes ou principes différents : le territoire politique et administratif national ou local, le territoire-enjeu, le territoire d'activités, le territoireprojet (Deffontaines et al., 2001). Cela s'exprime souvent dans le cadre de dispositifs de coordination, d'apprentissage et de régulation où interviennent des acteurs individuels, collectifs et publics, souvent en situation d'asymétrie d'information et de pouvoir. Le territoire constitué par l'action collective devient alors, pour certains auteurs (D'Aquino, 2002), un espace politique de débat permettant la confrontation entre société civile et institutions publiques. Il n'y a alors qu'un pas à faire pour voir se cristalliser dans ce mot de nombreuses propositions alternatives d'action cherchant à pallier les défaillances de l'État ou du marché. Le territoire devient le creuset des démarches participatives, contrastant avec les usages antérieurs du terme, faisant de lui un objet et un objectif de contrôle et de domination de la part du pouvoir politique central. Dans un contexte de décentralisation, il devient même le fer de lance d'une redécouverte des espaces infra-nationaux, visant à stimuler les initiatives d'acteurs privés, à garantir la fourniture ou la gestion de biens publics et à résoudre de nouveaux problèmes.

Dans ce mouvement des idées, comment et pourquoi les agronomes se sont-ils emparés du terme territoire, déjà réinvesti par les sciences humaines dans les années 1980 (Lévy et Lussault, 2003)? Nous tenterons, au cours du texte qui suit, de répondre à cette question en identifiant dans un premier temps les racines et les évolutions intellectuelles qui ont facilité ce chemin ${ }^{1}$. Nous identifierons ensuite des ensembles distincts de pratiques pour traiter et agir sur les territoires. Nous mobiliserons pour cela un ensemble d'expériences conduites dans les régions tropicales par les équipes de recherche du Centre de coopération internationale en recherche agronomique pour le développement (Cirad). Ces exemples ne prétendent pas à l'exhaustivité. Ils servent de support à l'élaboration d'un cadre d'analyse des pratiques $\mathrm{d}^{\prime}$ agronomes ${ }^{2}$.

\section{Comment les agronomes en sont-ils venus à s'intéresser à l'espace, puis au territoire?}

En 2000, la prise en compte «des échelles d'espace et de temps » peut paraître évidente (Malézieux et Trébuil, 2000). Il n'en n'a pas toujours été ainsi. S'intéresser à l'espace n'est certes pas nouveau pour les agronomes actuels, ni même pour leurs ancêtres romains (Hénin, 2001), mais l'espace reste alors un cadre dans lequel s'élabore une production diverse et variable. Pour décrire la variabilité spatiale des rendements culturaux, les agronomes conduisent depuis plusieurs décennies des études régionales de potentialités, élaborent des cartes, des zonages.

Les limites rencontrées dans l'exercice de leur métier les amènent par la suite à considérer de nouveaux objets d'analyse et à développer de nouvelles pratiques (Deffontaines, 1998). Ces évolutions s'opèrent via des ruptures : au gré des analyses critiques conduites au sein de la discipline, sous la houlette de praticiens confrontés à des questions posées par le monde professionnel ou la société. Depuis les années 1950, ces réflexions critiques sont essentiellement de trois ordres.

\footnotetext{
${ }^{1}$ Cet article est issu d'une communication présentée lors des seconds Entretiens du Pradel qui se sont déroulés les 12 et 13 septembre 2002 sur le thème "Agronomes et Territoires". À l'époque, l'auteur était directeur adjoint chargé des Affaires scientifiques du département Territoires, Environnement et Acteurs (TERA) du Cirad.

2 Nous nous intéressons au territoire comme catégorie d'analyse et d'action, et non au développement territorial comme finalité. Les pratiques centrées sur la conception et l'expérimentation de techniques, dont on peut assumer qu'elles aient toutes comme finalité de contribuer au développement territorial, ne seront pas discutées ici.
} 
Elles sont d'abord liées aux questions posées en matière de diffusion du progrès technique. Dans les pays tropicaux, il s'agissait, dès les années 1960 et 1970, de favoriser et d'accélérer le changement technique dans des agricultures jugées archaïques par des agronomes formés au Nord. Ces réflexions ont culminé en 1978 avec le colloque de Ouagadougou, Maîtrise de l'espace agraire et développement en Afrique tropicale, organisé par des géographes de l'ORSTOM, au cours duquel on a assisté à la réhabilitation de la variabilité et de l'efficacité de certaines agricultures paysannes africaines (ORSTOM, 1979). Dans les régions tempérées, les années 1970 voient émerger des « résistances » inattendues à l'adoption de nouvelles techniques, y compris celles permettant une augmentation du rendement et dont l'intérêt économique semblait jusque-là synonyme de diffusion rapide. Le contexte économique autorisait alors les agronomes à ne pas trop se soucier des objectifs et des contraintes des agriculteurs. Ces réflexions s'accompagnent bientôt d'une remise en cause d'un modèle unique de développement dit " productiviste » et essentiellement centré sur l'adoption de nouvelles techniques.

Elles sont ensuite mues par le souci qu'expriment la discipline et ses représentants d'une science finalisée et d'une utilité sociale. On parlera rapidement d'aide à la décision, sans que, pour autant, ce terme constitue dans un premier temps une question ou un objet de recherche. Le statut d'ingénieur acquis par l'étudiant, une formation organisée hors de l'Université, l'engagement dans l'action des praticiens, voire de certains chercheurs, y contribuent grandement mais sont aussi sources de « tension entre approfondissements disciplinaires et intégration des connaissances dans des cadres qu'il faut sans arrêt reconstruire » (Hervieu, 2001).

Elles sont enfin vivement encouragées par l'émergence de nouvelles préoccupations et questions et par l'expression par la société ou certaines catégories d'acteurs de nouvelles exigences (Papy, 2001). Dès les années 1970, les bouleversements scientifiques et sociaux ont réactivé la «question du sens d'une science de l'agriculture, de ses fondements, de ses liens avec d'autres disciplines, avec le milieu agricole et la société » (Denis, 2001). L'actualité plus récente met en exergue les préoccupations liées à la qualité et à la sécurité des produits, aux problèmes d'environnement, à la reconnaissance du caractère multifonctionnel des agricultures et des espaces ruraux. Ce dernier point oblige, par exemple, à poser un regard différent sur les règles de décision des agriculteurs, sur les critères d'évaluation des performances techniques et économiques des exploitants et du secteur agricole, et, partant, à élaborer de nouveaux concepts, méthodes et outils pour cela. Le champ de l'agronomie ne souhaite pas s'affranchir d'une telle rénovation.

$\mathrm{Au}$-delà de questions communes dans les pays du Nord et du Sud, certaines spécificités ont incité les agronomes tropicalistes, plus que leurs homologues « tempérés » peut-être, à s'intéresser à l'espace et aux processus sociaux de coordination à l'échelle territoriale (Eldin et Milleville, 1989 ; Biarnès, 1998). Il s'agit en particulier de l'importance des incertitudes et des conflits liés aux processus d'appropriation et d'usage des ressources foncières. Par ailleurs, la rapidité et la violence des dérégulations et des recompositions économiques, politiques et réglementaires se traduisent par une intense différenciation spatiale et une mobilité exacerbée. Sans aucun doute, une rencontre précoce avec les géographes y a également contribué.

Les années 1970 sont traversées par l'influence de la pensée systémique. Cette marque n'est pas propre aux sciences agronomiques, mais celles-ci y sont particulièrement sensibles. On voit là une manière de se saisir et de répondre à de nouvelles questions. Dans un premier temps, il s'agit de reconnaître et de caractériser la diversité : celle des sols, puis des exploitations. Les approches système sont rapidement mobilisées pour rendre intelligibles la diversité et la complexité des faits de l'agriculture, à l'aide de nouveaux concepts : système de culture, d'élevage, de production, système agraire, etc. Le but est de faciliter le "transfert de technologies » en tenant compte des contraintes auxquelles les agriculteurs font face. Dans la continuité des réflexions engagées par Hénin et s'inspirant du modèle canonique de Le Moigne (1990), les agronomes se penchent dans les années 1980 sur les systèmes décisionnels et de pilotage des agriculteurs (Duru et al., 1988; Sebillotte et Soler, 1990). Ils élaborent de nouveaux concepts, de nouvelles méthodes, reconnaissent de nouveaux objets de recherche, à l'exemple des pratiques mises en œuvre par les agriculteurs (Landais et Deffontaines, 1990). Ils ne s'intéressent plus seulement aux modèles d'élaboration des rendements, ce qui retenait l'essentiel de l'attention depuis la Seconde Guerre mondiale, mais aussi aux modèles d'élaboration des décisions. Ce mouvement s'accompagne d'une rénovation des modalités de conception des dispositifs partenariaux au sein desquels la recherche intervient : recherche-développement, recherche-action inspirée des sciences de l'éducation et de la médecine (Albaladejo et Casabianca, 1997), recherche-intervention puisant ses fondements dans les sciences de gestion.

$\mathrm{Au}$ cours de ces évolutions, la référence à l'espace gagne droit de cité : il est pris en compte pour penser et interpréter la diversité des situations, comme cadre d'action spécifique de la localité, comme facteur essentiel dans l'élaboration des choix de gestion de l'exploitant agricole et non pas uniquement des assolements. Certains termes porteurs de la dimension spatiale de l'activité agricole apparaissent explicitement dans la définition des nouveaux concepts : celui de système agraire reconnaît la région comme niveau d'organisation (Jouve et Clouet, 1984; Mazoyer et Roudard, 1997), celui de système 
d'élevage, le territoire comme l'un de ses trois pôles constitutifs (Lhoste, 1984).

Les agronomes tropicalistes ne sont pas en reste. Les spécificités propres au théâtre de leurs activités enrichissent les réponses apportées aux questions communes, confirmant l'intérêt d'une "transgression géographique » des dispositifs de recherche souhaitée par Sautter (1993) et matérialisée par le volume collectif Le Développement rural en questions (Blanc-Pamard et al., 1984). Un cas reste particulier, celui de la gestion des terroirs (Teyssier, 1995), thème développé dans les années 1980, peut-être précurseur des réflexions actuelles concernant l'implication des agronomes dans le champ de l'environnement. Les actions visaient à promouvoir des aménagements pour garantir le maintien de la «capacité de production » des ressources naturelles et apporter des solutions pour lutter collectivement, à l'échelle des finages (improprement baptisés terroirs), contre leur "dégradation", réelle ou plus souvent déclarée par les experts. D'intenses fécondations s'opèrent alors avec les géographes ruralistes, qui avaient pris quelques décennies d'avance en s'intéressant aux logiques paysannes et se retrouvent surpris et ravis de voir les agronomes sortir de leurs stations et prendre en compte l'espace (Marchal, 1991), et de participer avec eux à des séminaires de recherche et à des formations doctorales (Raison, 1993). C'est en particulier le cas du séminaire « Dynamique des systèmes agraires », organisé de 1983 à 1995 par des géographes du Centre d'études africaines et des chercheurs de l'ORSTOM, et consacré aux sociétés et aux espaces ruraux dans les pays du tiers-monde. Il jette les bases d'une connaissance plus approfondie du monde rural en faisant appel à des chercheurs et à des praticiens appartenant à diverses institutions (Blanc-Pamard et Lericollais, 1985; Blanc-Pamard et Boutrais, 1994; Blanc-Pamard et Cambrézy, 1995 ; Blanc-Pamard et Boutrais, 1997).

Au début des années 1990, certains agronomes commencent à parler de changement d'échelle (Caron et al., 1996). Ils sont motivés par les limites rencontrées dans le cadre d'une action locale et se proposent d'agir, à d'autres niveaux d'organisation, sur un ensemble de facteurs tels que le fonctionnement des marchés, la législation foncière, les politiques agricoles, l'aménagement du territoire, etc., à partir de connaissances acquises localement à propos du fait technique. Ils souhaitent ainsi améliorer les termes de leur action pour le développement et en élargir géographiquement et thématiquement le champ. Il s'agit de sortir du local pour mieux agir au local. À l'expression «changement d'échelle » est rapidement préférée celle d' ' intégration entre niveaux d'analyse et d'action » (Caron, 1998), qui rend mieux compte des interférences entre processus se déroulant selon des échelles spatiales et temporelles différentes. De nouvelles questions sont formulées, de nouveaux objets identifiés, ainsi que les manières de les traiter. Tonneau et al. (1997) proposent, par exemple, une recherche par analyse spatiale pour identifier les espaces économiques, historiques et géographiques de l'agriculture familiale dans le Nordeste du Brésil.

$\mathrm{Au}$ cours de la dernière décennie, la référence à l'espace, plus souvent à la région ou au territoire, marque ainsi de son empreinte les institutions de recherche agronomique (Encadré 1 ).

Les années 1970-1990 sont ainsi marquées par la mobilisation par les agronomes d'autres disciplines, en particulier les sciences humaines : sociologie, économie, sciences de gestion et de la cognition, géographie, anthropologie, etc. Certains se baptisent même agroéconomiste, agro-sociologue, géo-agronome, etc. Ces passeurs de frontière vont chercher chez l'autre ce qui permet de répondre à de nouvelles questions ou de dépasser la critique. Mais cet enrichissement s'accompagne $\mathrm{d}^{\prime}$ un double malaise. Le premier consiste en la difficile reconstitution du champ en expansion de l'agronomie, posant des problèmes d'identité ${ }^{3}$, de frontières et de reconnaissance au sein de la discipline pour ceux qui la tirent vers de nouveaux horizons. L'émergence de courants de pensée et d'écoles s'accompagne d'un risque de scission (Académie d'agriculture de France, 2001 ; Denis, 2001). La segmentation croissante du champ disciplinaire contraste avec la nécessaire intégration des connaissances d'une discipline finalisée. Le second malaise tient aux relations entretenues avec d'autres disciplines dans le cadre de dispositifs pluridisciplinaires et à la réticence qu'exprime l'agronome à être convoqué par d'autres au titre de son expertise. Tout cela pose, entre autres, la question de l'évaluation de telles activités (Sebillotte, 2001b ; Hubert et Bonnemaire, 2000).

Cette période est enfin marquée par le développement spectaculaire d'outils informatiques d'analyse et de représentation spatiale des données, à l'exemple des systèmes d'information géographique (SIG), qui contribuent en retour à un renouvellement de la prise en compte de l'espace par les agronomes.

Chemin faisant, les agronomes se sont donc intéressés à l'espace, parfois au territoire. Ce fait s'est traduit par la reconnaissance et la prise en compte de nouveaux niveaux d'organisation et catégories d'analyse. On peut identifier différentes familles d'agronomes, ayant chacune emprunté à des courants de pensée et des disciplines spécifiques, suivi des trajectoires particulières, alors qu'elles forgeaient dans le même temps leurs spécificités et leur singularité.

\footnotetext{
3 Une agronomie au sens large correspondant à « une partie ou la totalité des sciences appliquées à l'agriculture » (Hénin et Sebillotte, 1996) ou une agronomie au sens strict définie comme écologie appliquée (Malézieux et Trébuil, 2000) et développant des « continuums » avec d'autres disciplines?
} 
Encadré 1. L'apparition de l'espace dans les institutions de recherche agronomique à partir des années 1990

Le Groupe consultatif pour la recherche agricole internationale propose le concept d'éco-régionalité. Le recours à ce terme vise à mieux programmer les activités et à mettre en place des dispositifs de recherche à des niveaux régionaux englobants en tenant compte de la diversité des situations agro-écologiques. Il est repris par des auteurs français (Manichon, 1996; Lhoste et al., 1998), mais inclut alors l'élaboration d'un diagnostic régional.

Le Cemagref crée un département Gestion des territoires, l'Engref, un département Aménagement du territoire et Développement local. À l'Inra, la Délégation permanente à l'agriculture, au développement et à la prospective (DADP) lance un programme «pour » et «sur» le développement régional, revendiquant une nouvelle forme d'implication du chercheur avec et en appui à des partenaires ayant des responsabilités régionales de développement (Sebillotte, 2000). La région est dans ce cas un territoire administratif doté de moyens d'intervention, mais l'articulation des actions à différents niveaux de l'organisation territoriale pose question (Sebillotte, 2001a). Dans son schéma directeur 1999-2003, le département Systèmes agraires et Développement (SAD) de l'Inra reconnaît l'axe «Développement local et territorialité » comme l'un de ses quatre champs thématiques.

Au Cirad, le département des Systèmes agraires (DSA), créé en 1984 et devenu Systèmes agro-alimentaires et ruraux (SAR) en 1992, fait place en 1998 au département Territoires, Environnement et Acteurs (TERA), s'inscrivant dans la continuité des travaux menés sur les systèmes de production, les systèmes agraires, la gestion des terroirs et des ressources renouvelables, le développement local. Sa création s'appuie sur la volonté « d'intégrer les exigences du développement durable, de répondre aux besoins des collectivités territoriales dans le cadre des politiques de décentralisation » (Tonneau et Pichot, 1999). Le passage du DSA à TERA, des systèmes agraires au développement régional, est justifié, entre autres, par la volonté de promouvoir l'implication des chercheurs dans les processus de transformation, à des échelles allant du local au régional. En 2002, le nouveau projet du département, comportant environ un tiers d'agronomes, propose, comme inflexion majeure, de substituer le terme « territorial » au terme « régional » et de reconnaître les dynamiques territoriales comme objet central d'investigation. Le recours à la notion de territoire vise à explorer et à donner sens aux relations complexes qui se tissent entre les acteurs, leurs organisations et leur environnement. Le terme «dynamique » est préféré à celui de «développement » pour rendre compte de processus qui procèdent le plus souvent par ruptures et adaptations plus ou moins viables et de l'implication de nombreuses catégories d'acteurs dans le changement.

\section{Différentes voies de prise en compte du territoire par les agronomes}

On peut différencier quatre manières de s'intéresser au territoire, selon les caractéristiques des objets territoriaux traités, les référents théoriques et disciplinaires mobilisés et les pratiques mises en œuvre, qu'elles soient de l'ordre de l'analyse ou de l'action.

La première voie correspond à une agronomie de l'écosystème régional. Elle vise à étudier les termes des relations climat-sol-peuplements végétaux soumis à l'action de l'homme au niveau d'une région donnée. Cette dernière est un espace administratif ou une unité écologique, et ne correspond donc pas nécessairement à un territoire au sens proposé ci-dessus. Cette pratique renoue avec les analyses régionales qui ont de tout temps intéressé les agronomes. L'objet ne se résume toutefois pas à la diversité spatiale des rendements. La région est considérée comme système et l'on cherche à caractériser les interactions biogéochimiques - en surface et en profondeur -, ou entre pratiques mises en œuvre et processus observés en des lieux différents. L'intégration de cinétiques diverses et interdépendantes pose souvent question. Cette agronomie est indispensable pour traiter des questions actuelles d'environnement. En effet, il n'y a pas, en général, coïncidence entre le niveau auquel est posée la question et les entités d'action, que ces dernières relèvent de catégories gérées par des individus et des institutions ou de formes territoriales construites socialement. Il s'agit d'identifier ce que les économistes appellent externalités, à savoir des effets désirables ou non générés par une dissociation spatiale et/ou temporelle entre niveaux d'action et d'accomplissement d'un processus particulier, et de résoudre les problèmes qu'elles posent. L'implication de l'agronome ne permet pas, seule, de résoudre le problème. Son analyse est néanmoins nécessaire pour élaborer des connaissances, des normes et des référentiels au niveau de concernement du problème. Dans le cas d'une recherche sur la modélisation des flux de biomasse et des transferts de fertilité appliquée au cas de la gestion des effluents d'élevage, Saint Macary et al. (à paraître) identifient, par exemple, les principaux puits et gisements d'azote à l'échelle de l'île de la Réunion. Cette connaissance vise à mieux caractériser les termes $\mathrm{d}^{\prime}$ un problème à résoudre et à concevoir ultérieurement des actions à mettre en place, aux niveaux de l'exploitation agricole, de collectifs (ex. : mise en place d'unités collectives de traitement) et public (élaboration de normes et de réglementations, aménagement du territoire, etc.). L'ambition est bien d'agir sur les processus biogéochimiques impliqués dans des problèmes d'environnement, et l'analyse mobilise pour cela les référents théoriques et méthodologiques de sciences de la nature. Elle s'appuie aussi sur une caractérisation des pratiques de production, élaborée par le recours à des sources d'information hétérogènes (dires d'experts et données statistiques).

La seconde voie est baptisée agronomie du fait technique à l'échelle territoriale. Elle se situe d'emblée en rupture avec la précédente en mobilisant des référents 
issus des sciences humaines. Elle concerne le pilotage et la gestion des agrosystèmes. Elle se fonde sur la possibilité de « rendre compréhensibles les pratiques agricoles en explicitant les interdépendances entre les systèmes de culture pratiqués et l'aménagement des territoires » (Papy, 2001). Elle s'intéresse à la constitution d'objets techniques à l'échelle territoriale, à savoir des dispositifs d'action élaborés socialement, mobilisés et pilotés par des organisations collectives. Elle vise à comprendre et à agir sur des systèmes techniques au travers de processus de coordination, d'apprentissage, de négociation et de régulation. Elle prend en compte le territoire et permet d'agir à ce niveau, mais ne le reconnaît pas comme objet d'analyse. Ce type d'approche a fait l'objet d'importants travaux depuis une vingtaine d'années au niveau de l'exploitation agricole. À l'exemple de l'analyse des relations entre processus décisionnels et biotechniques mis en jeu dans le fonctionnement des systèmes techniques (Landais et Bonnemaire, 1996), bon nombre de cadres méthodologiques ont été ainsi élaborés. Ces derniers facilitent le renouvellement de l'appréhension $\mathrm{du}$ fait technique au regard des évolutions récentes du contexte agricole et rural et de la reconnaissance de la multifonctionnalité de l'agriculture. Ils permettent d'intervenir en appui au changement technique dans le cadre de réseaux territoriaux. L'intérêt pour l'innovation et le changement technique place l'agronome en position de contribuer à l'intégration de connaissances et d'actions concernant des champs disciplinaires et des formes spatiales et temporelles d'organisation distinctes. Au cœur du Nordeste brésilien, dans une zone où l'existence de parcours collectifs est menacée par des processus conflictuels d'enclosure, Sabourin et al. (1997) montrent comment des communautés d'agriculteurs familiaux se sont organisées pour mettre en œuvre des stratégies collectives de gestion de la ressource. L'étude s'appuie sur une caractérisation des pratiques individuelles et collectives des acteurs en matière de gestion du patrimoine foncier, $\mathrm{du}$ troupeau et de l'espace, et de mise en œuvre de techniques différenciées de clôture. Elle montre comment les communautés ont créé de nouvelles institutions et règles d'appropriation et d'usage du foncier, reposant sur des actions d'aménagement de l'espace et contribuant, en retour, à une évolution des techniques d'élevage. Des exemples similaires pourraient être présentés, concernant la gestion de la production au sein de périmètres irrigués au Sénégal (Le Gal et Papy, 1998), l'organisation de bassins de collecte des produits agricoles ou la construction sociale de la qualité des produits dans le cadre de systèmes agroalimentaires localisés (Requier-Desjardins et al., à paraître).

La troisième voie est celle de l'agronomie territoriale. Elle analyse la contribution du fait technique, qu'elle considère processeur de changement, à la production de territoires, érigée au rang de catégorie d'analyse.
Elle s'inscrit dans un courant de réflexion dépassant l'agronomie et se reconnaissant dans le terme d'ingénierie des territoires, à savoir « l'ensemble des méthodes et des techniques permettant d'analyser les territoires et d'intervenir sur leurs contenus » (Mégie, 2001). Un cas particulier concerne la production de paysage (Candau et Le Floch, 2001). Caron et Hubert (2000) ont montré, dans le cas du Nordeste brésilien, que les systèmes d'élevage ne sont pas uniquement « révélateurs » des espaces ruraux, au sens où ils sont marqués par les spécificités de leur environnement. Ils peuvent également être considérés comme "organisateurs ». Avec l'évolution des activités, l'espace acquiert de nouvelles caractéristiques, il est le siège de nouveaux usages. Les fonctions qui lui sont attribuées changent de nature avec les transformations qualitatives des ressources opérées pour satisfaire aux besoins de l'élevage. Les activités d'élevage donnent naissance à de nouvelles formes d'organisation locale, comme, par exemple, celles de gestion des communs. Approprier des ressources pastorales, changer d'espèce animale, de race ou de production créent des institutions, des règles d'action, en un mot, produisent de nouveaux territoires. Ainsi, une telle agronomie contribue à l'identification de formes territoriales et de niveaux fonctionnels pertinents pour la compréhension des dynamiques et pour l'action qui, loin d'être donnés a priori, font sens et donnent un sens nouveau au comportement des acteurs (Caron, 1998). Par ailleurs, les différents niveaux et formes d'organisation identifiés au cours de telles études coïncident rarement. Un engagement dans les dynamiques de changement peut alors consister à favoriser la coordination entre organisations et acteurs dont les principes d'action résident à différents niveaux. $C^{\prime}$ est bien de cela qu'il s'agit lorsqu'on en vient à découpler les fonctions assurées par l'activité agricole, selon les niveaux concernés, dans une optique de développement durable.

Enfin, la dernière voie, pouvant recouvrir en partie les formes précédentes et les mobilisant, est celle visant à élaborer des cadres d'analyse régionale, à identifier des niveaux d'organisation ayant du sens, à formuler des questions traitables scientifiquement (Sebillotte, 2001a; Tonneau et Pichot, 1999; Tonneau et al., 1997). Landais et Bonnemaire (1996) affirment que « le zootechnicien doit être capable d'analyser les nouvelles fonctions de l'élevage et de les resituer dans un ensemble complexe de finalités et d'interactions ». Cette implication est courante chez les praticiens. Elle reste rare chez les chercheurs et questionne les articulations entre action, connaissances et modes d'acquisition de ces connaissances. La tentation est parfois grande de baptiser généraliste un tel agronome. Ce qualificatif, outre la raillerie de laisser croire qu'il ne sache rien sur rien, pose des problèmes de collaboration avec les pairs de la discipline, pouvant refuser d'être convoqués au titre de leur expertise. 
Pourtant, le développement de telles compétences « d'intégrateur territorial » est bien nécessaire pour limiter l'étanchéité entre familles d'agronomes, voire l'éclatement entre, d'un côté, des "gestionnaires de l'espace rural », de l'autre, des « ingénieurs biotechnologistes » (Denis, 2001), pour éviter que chacun fasse «son métier honnêtement au regard des exigences immédiates, de manière fautive par rapport à l'avenir " (Sebillotte, 2001a). Encore faut-il reconnaître et stabiliser un certain nombre de questions à traiter, d'objets et de manières de les traiter, propres à l'agronomie - et se différenciant ainsi de ce qui peut se faire au sein d'autres disciplines. Il s'agit en premier lieu de l'identification et de la hiérarchisation de moyens d'action faisant le lien entre processus biophysiques, procédures techniques et dynamiques sociales. Encore faut-il transformer en énoncés enseignables un corpus de connaissances, de méthodes et d'outils en général construits ou acquis via l'expertise et l'expérience et trop peu formalisés.

\section{Conclusion}

Les questions soulevées par les mutations des sociétés et du monde agricole, mais aussi l'évolution des métiers et du monde scientifiques militent pour une approche adéquate du territoire par les agronomes. En retour, nous avons vu, à l'aide d'exemples, comment le seul fait de s'y intéresser amène les chercheurs à formuler et traiter de nouvelles questions.

Dans le prolongement des recherches sur les systèmes agraires, des travaux ont effectivement été entrepris au cours des dernières années pour identifier et qualifier le territoire et son rôle et agir en appui aux dynamiques territoriales. Ce qui «fait territoire » et la manière de le prendre en compte ne font pas unanimité. Nous avons ainsi identifié des pratiques de recherche différenciées, le territoire faisant dans chaque cas référence à un objet distinct : un niveau d'organisation, un cadre d'analyse, un objet construit, une ressource, voire un facteur de changement. Derrière chaque acception, se cachent des référents théoriques, souvent constitués en mobilisant d'autres disciplines.

Assumer la diversité et la pluralité des pensées, des méthodes et des métiers ne va pas non plus de soi et peut prêter à équivoque, voire à polémique, entre représentants d'une agronomie de l'écosystème régional s'adossant aux sciences de la nature et les autres courants identifiés et ayant tous fait de larges emprunts aux sciences humaines, en particulier à la géographie.

Comme le rappelle B. Hervieu (2001), l'enjeu est de taille et vise à inscrire ces communautés « dans la longue tradition ouverte par Olivier de Serres, faisant de l'agronomie une science d'intégration et de synthèse et de l'agriculture un enjeu de société ». Le territoire, objet hybride des sciences de la nature et de l'homme, offre un support privilégié pour permettre à l'agronomie de revendiquer une telle vocation. Il pourrait, en conséquence, constituer un objet - parmi d'autres - permettant de renouer avec un dessein brouillé par le risque, souligné lors des premiers Entretiens du Pradel (Académie d'agriculture de France, 2001), d'éclatement au sein de la discipline entre "gestionnaires de l'espace rural » et «ingénieurs biotechnologistes ». Ceci requiert un projet permettant aux agronomes de se situer, de communiquer, d'enseigner et de mobiliser les moyens pour œuvrer en synergie autour de problèmes concrets de développement territorial et d'affirmer la légitimité d'un projet scientifique porté par des agronomes « intégrateurs territoriaux ».

\section{Références}

Académie d'agriculture de France, 2001. Les Entretiens du Pradel, $1^{r e}$ édition. Autour d'Olivier de Serres : pratiques agricoles et pensée agronomique, Comptes rendus de l'Académie d'agriculture de France, 87, 4.

Albaladejo, C., Casabianca, F., 1997. La Recherche-action : ambitions, pratiques, débats, Versailles, INRA Éditions.

Aquino, P. d', 2002. Le local entre espace et pouvoir : pour une planification territoriale ascendante, L'Espace géographique, 1, 3-22.

Biarnès, A. (Ed.), 1998. La Conduite du champ cultivé : points de vue d'agronomes, Paris, ORSTOM.

Blanc-Pamard, C., Bonnemaison, J., Boutrais, J., 1984. Le Développement rural en questions, Bondy, ORSTOM.

Blanc-Pamard, C., Lericollais, A. (Eds), 1985. Dynamique des systèmes agraires. À travers champs, agronomes et géographes, Paris, ORSTOM.

Blanc-Pamard, C., Boutrais, J. (Eds), 1994. Dynamique des systèmes agraires. À la croisée des parcours : pasteurs, éleveurs, cultivateurs, Bondy, ORSTOM.

Blanc-Pamard, C., Cambrézy, L. (Eds), 1995. Dynamique des systèmes agraires. Terre, terroir, territoire : les tensions foncières, Bondy, ORSTOM.

Blanc-Pamard, C., Boutrais, J., 1997. Dynamiques des systèmes agraires. Thèmes et variations : nouvelles recherches rurales au Sud, Paris, ORSTOM.

Bonnemaison, J. (établi par Lasseur, M., Thibault, C.), 2000. La Géographie culturelle : cours de l'Université Paris IV-Sorbonne, 1994-1997, Paris, CTHS.

Brunet, R., Ferras, R., Théry, H., 1992 (2 éd.). Les Mots de la géographie : dictionnaire critique, Montpellier/Paris, Reclus/La Documentation française.

Camagni, R., 1991. Local «milieu», uncertainty and innovation networks: towards a new dynamic theory of economic space, in Camagni, R. (Ed.), Innovation Networks: Spatial Perspectives, London, Belhaven Press.

Candau, J., Le Floch, S., 2001. Le paysage comme catégorie d'action publique, Natures Sciences Sociétés, 10, 2, 59-65.

Caron, P., Tonneau, J.-P., Sabourin, É., 1996. Planification locale et régionale : enjeux et limites. Le cas du Brésil Nordeste, in Globalisation, Competitioness and Human Security: Challenge for Development Policy and Institutional Change, 
VIIIth Conference of European Association of Development, Research and Training Institutes, Vienne, EADI, 11-14 september.

Caron, P., 1998. Espace, élevage et dynamique du changement. Analyse, niveaux d'organisation et action. Le cas du Nordeste semi-aride $d u$ Brésil. Thèse de doctorat en géographie, Université Paris X-Nanterre.

Caron, P., Hubert, B., 2000. De l'analyse des pratiques à la construction d'un modèle d'évolution des systèmes d'élevage : application à la région Nordeste du Brésil, Élev. Méd. Vét. Pays Trop., 53, 1, 37-53.

Deffontaines, J.-P., 1998. Les Sentiers d'un géoagronome, Paris, Arguments.

Deffontaines, J.-P., Marcelpoil, E., Moquay, P., 2001. Le développement territorial : une diversité d'interprétations, in Lardon, S., Maurel, P., Piveteau, V. (Eds), Représentations spatiales et développement territorial, Paris, Hermès Sciences Publications.

Denis, G., 2001. Du physicien agriculteur du dix-huitième à l'agronome des dix-neuvième et vingtième siècles : mise en place d'un champ de recherche et d'enseignement, Comptes rendus de l'Académie d'agriculture de France, 87, 4, 81-103.

Duru, M., Papy, F., Soler, L.G., 1988. Le concept de modèle général et l'analyse du fonctionnement de l'exploitation agricole, Comptes rendus de l'Académie d'agriculture de France, 74 , 4, 81-93.

Eldin, M., Milleville, P. (Eds), 1989. Le Risque en agriculture, Paris, ORSTOM.

Hénin, S., 2001. Olivier de Serres. « Le théâtre d'agriculture et mesnage des champs ", une étape clé entre les agronomes latins et la mise en place de la pensée objective moderne, Comptes rendus de l'Académie d'agriculture de France, 87, 4, 23-29.

Hénin, S., Sebillotte, M., 1996. Agronomie, in Encyclopedia Universalis, 618-622.

Hervieu, B., 2001. Olivier de Serres : actualité d'un hommage, Comptes rendus de l'Académie d'agriculture de France, 87, 4, 13-16.

Hubert, B., Bonnemaire, J., 2000. La construction des objets dans la recherche interdisciplinaire finalisée : de nouvelles exigences pour l'évaluation, Natures Sciences Sociétés, 8, 3, 5-19.

Jouve, P., Clouet, Y., 1984. La fonction diagnostic appliquée à l'étude des systèmes agraires, Les Cahiers de la recherchedéveloppement, 3-4, 5-9.

Landais, É., Deffontaines, J.-P., 1990. Les pratiques des agriculteurs : point de vue sur un courant nouveau de la recherche agronomique, in Brossier, J., Vissac, B., Le Moigne, J.-L., Modélisation systémique et systèmes agraires : décision et organisation, Actes du séminaire du Département de recherches sur les systèmes agraires et le développement (SAD), SaintMaximin (2 et 3 mars 1989), Paris, INRA, 31-64.

Landais, É., Bonnemaire, J., 1996. La zootechnie, art ou science? Entre nature et société, l'histoire exemplaire d'une discipline finalisée, in Le Courrier de l'environnement de l'INRA, 27, 23-44.

Le Gal, P.-Y., Papy, F., 1998. Coordination processes in a collectively managed cropping system: double cropping of irrigated rice in Senegal, Agricultural systems, 57, 135-159.

Le Moigne, J.-L., 1990. La Modélisation des systèmes complexes, Paris, Dunod.
Lévy, J., Lussault, M. (Eds), 2003. Dictionnaire de la géographie et de l'espace des sociétés, Paris, Belin.

Lhoste, P., 1984. Le diagnostic de système d'élevage, Les Cahiers de la recherche-développement, 3-4, 84-88.

Lhoste, P., Tonneau, J.-P., Trébuil, G., 1998. Recherche écorégionale et développement régional : enjeux, démarche et outils, Les Cahiers de la recherche-développement, 45, 7-36.

Malézieux, É., Trébuil, G., 2000. L'Agronomie et la gestion de l'environnement et des ressources naturelles au Cirad : réflexions, propositions, éléments de prospective, Montpellier, CIRAD-AGER.

Manichon, H., 1996. L'Écorégionalité dans la recherche pour le développement : propositions de la recherche française, Montpellier, CIRAD.

Marchal, J.-Y., 1991. Quand les agronomes s'en vont aux champs, L'Espace géographique, 3, 214-221.

Mazoyer, M., Roudard, L., 1997. Histoire des agricultures du monde, Paris, Le Seuil.

Mégie, G., 2001. L'ingénierie des territoires, Natures Sciences Sociétés, 10, suppl. 1, 92.

ORSTOM, 1979. Maîtrise de l'espace agraire et développement en Afrique tropicale : logique paysanne et rationalité technique, Actes du colloque de Ouagadougou (4-8 décembre 1978), Paris, ORSTOM.

Papy, F., 2001. Pour une théorie du ménage des champs : l'agronomie des territoires, Comptes rendus de l'Académie d'agriculture de France, 87, 4, 139-149.

Raison, J.-P., 1993. Trente ans, trois phases de la géographie rurale dans les pays tropicaux, in Choquet, C., Dollfus, O., Le Roy, É., Vernières, M., 1993. État des savoirs sur le développement. Trois décennies de sciences sociales en langue française, Paris, Karthala, 135-159.

Requier-Desjardins, D., Boucher, F., Cerdan, C., à paraître. Globalization, competitive advantages and the evolution of production systems: rural food processing and localized agri-food systems in Latin-American countries. Entrepreneurship \& Regional Development.

Sabourin, É., Caron, P., Silva, P.C.G. da, 1997. Enjeux fonciers et gestion des communs dans le Nordeste du Brésil : le cas des vaines pâtures dans la région de Massaroca-Bahia, Les Cahiers de la recherche-développement, 42, 5-27.

Saint Macary, H., Médoc, J.-M., Chabalier, P.-F., à paraître. Systèmes de culture de la Réunion. Typologie, spatialisation et éléments pour un référentiel, in Guerrin, F., Paillat, J.-M. (Eds), Modélisation des flux de biomasse et des transferts de fertilité : cas de la gestion des effluents d'élevage à l'île de la Réunion, Actes du séminaire des 19-20 juin 2002, Montpellier, CIRAD.

Sautter, G., 1993. Le temps des méthodes, in Parcours d'un géographe. Des paysages aux ethnies, de la brousse à la ville, de l'Afrique au monde, 2 vol., Paris, Arguments.

Sebillotte, M., 2000. Des recherches pour le développement local. Partenariat et transdisciplinarité, Revue d'économie régionale et urbaine, 3, 535-556.

Sebillotte, M., 2001a. Des recherches en partenariat «pour» et "sur» le développement régional. Ambitions et questions, Natures Sciences Sociétés, 9, 3, 5-7. 
Sebillotte, M., 2001b. Les fondements épistémologiques de l'évaluation des recherches tournées vers l'action, Natures Sciences Sociétés, 9, 3, 8-15.

Sebillotte, M., Soler, L.G., 1990. Les processus de décision des agriculteurs, in Brossier, J., Vissac, B., Le Moigne, J.-L., Modélisation systémique et systèmes agraires : décision et organisation, Actes du séminaire du Département de recherches sur les systèmes agraires et le développement (SAD), Saint-Maximin (2 et 3 mars 1989), Paris, INRA, 93-118.

Reçu le 8 mars 2004. Accepté le 3 mars 2005.
Teyssier, A., 1995. La gestion de terroir. A review of a francophone concept and a case study of its application, Rural Extension Bulletin, 7, 43-48.

Tonneau, J.-P., Pichot, J.-P., 1999. Une recherche pour le développement régional : la création du département Territoires, Environnement et Acteurs au Cirad, Les Cahiers de la recherche-développement, 45, 37-50.

Tonneau, J.-P., Clouet, Y., Caron, P., 1997. L'agriculture familiale au Nordeste (Brésil). Une recherche par analyses spatiales, Natures Sciences Sociétés, 5, 3, 39-49.

To access this journal online: www.edpsciences.org 